UR-1274

ER-40425-260

\title{
SELF-DUALITY AND THE KdV HIERARCHY
}

\author{
Ashok Das \\ and \\ C. A. P. Galvão * \\ Department of Physics and Astronomy \\ University of Rochester \\ Rochester, NY 14627, USA
}

\begin{abstract}
$\underline{\text { Abstract }}$
We derive the entire KdV hierarchy as well as the recursion relations from the selfduality condition on gauge fields in four dimensions.
\end{abstract}

* Permanent address: CBPF, Dept. of Fields and Particles, Rua Dr Xavier Sigaud 150, Urca, 22290 Rio de Janeiro, Brazil 


\section{Introduction:}

Most of the integrable equations in $1+1$ dimensions [1-2] such as the KdV (Kortweg -deVries) equation, the Sine-Gordon equation, the nonlinear Schrödinger equation, can be derived from $\mathrm{SL}(2, \mathbf{R})$ valued gauge fields in four dimensions by requiring their field strengths to be self-dual [3-5]. More recently, even the $2+1$ dimensional KP (KadomtsevPetviashvili) equation [6] has been derived from a self-duality condition imposed on $\operatorname{SL}(2, \mathbf{R})$ valued gauge fields in six dimensions [7]. The solutions to the self-duality conditions are extremely restrictive and even though individual integrable equations (e.g. KdV) can be obtained from these conditions, it has proved infinitely difficult to obtain from these the hierarchy of equations associated with a given equation.

In this letter, we show how the entire KdV hierarchy of equations along with the appropriate recursion relations can be obtained from the self-duality condition on gauge fields in four dimensions. In sec. II we give a derivation of the KdV hierarchy based on an Abelian zero curvature condition. We would like to emphasize that the standard zero curvature derivation of the $\mathrm{KdV}$ hierarchy $[8,2]$ uses the Lie group $\mathrm{SL}(2, \mathbf{R})$. This derivation based on the Abelian symmetry is, therefore, new and is useful in the discussion of the selfduality condition. In sec. III we show how the KdV hierarchy can be obtained from the self-duality condition imposed on Abelian gauge fields in four dimensions. In sec. IV we derive the $\mathrm{KdV}$ hierarchy from $\mathrm{SL}(2, \mathbf{R})$ valued gauge fields in four dimensions by imposing the self-duality condition. We present our conclusions in sec. V.

\section{KdV Hierarchy:}

The derivation of the KdV hierarchy from a zero curvature condition associated with the group $\mathrm{SL}(2, \mathbf{R})$ in $1+1$ dimension is quite standard [8,2]. In this section, we show how the hierarchy can be obtained from a vanishing field strength associated with an Abelian gauge field in $1+1$ dimension. Let $A_{\mu}(x), \mu=0,1$, denote an Abelian gauge field in $1+1$ 
dimension. Then, the zero curvature condition reads

$$
\begin{gathered}
F_{01}=\partial_{0} A_{1}-\partial_{1} A_{0}=0 \\
\text { or, } \quad \partial_{0} A_{1}=\partial_{1} A_{0}
\end{gathered}
$$

where $\partial_{0}=\frac{\partial}{\partial t}$ and $\partial_{1}=\frac{\partial}{\partial x}=\partial$. It is clear that if we identify

$$
\begin{aligned}
& A_{1}(x, t)=u(x, t) \\
& A_{0}(x, t)=\frac{\delta H_{n}}{\delta u(x, t)}
\end{aligned}
$$

where $u(x, t)$ is the dynamical variable of the $\mathrm{KdV}$ hierarchy and $H_{n}[u]$ is the nth conserved charge, then the zero curvature condition of Eq. (1) would take the form

$$
\frac{\partial u}{\partial t}=\frac{\partial}{\partial x} \frac{\delta H_{n}}{\delta u(x, t)}
$$

which we recognize to be the nth order equation in the KdV hierarchy.

Let us further note that if we choose

$$
\begin{aligned}
& A_{1}(x, t)=u(x, t) \\
& A_{0}(x, t)=\left(-2 \lambda^{2}+\frac{1}{2} \partial^{-1}\left(\partial^{3}+2(2 u+u \partial)\right)\right) C(u)
\end{aligned}
$$

where $\lambda$ is an arbitrary constant parameter (commonly known as the spectral parameter), $\partial^{-1}$ is the formal inverse of the derivative operator (can be thought of as a space integration) and $C(u)$ is a functional of the dynamical variable $u(x, t)$, then the zero curvature condition would give

$$
\begin{aligned}
\partial_{0} A_{1} & =\partial_{1} A_{0}=\partial A_{0} \\
\text { or, } \quad \frac{\partial u}{\partial t} & =-2 \lambda^{2} \partial C(u)+\frac{1}{2}\left(\partial^{3}+2(\partial u+u \partial)\right) C(u)
\end{aligned}
$$

The derivation of the KdV hierarchy as well as the recursion relations is now standard (see for example, ref. 2 p137-138). We note here that it is the biHamiltonian structure of the KdV hierarchy which leads to the derivation of these equations from two distinct zero curvature conditions based on the groups $\mathrm{U}(1)$ and $\mathrm{SL}(2, \mathbf{R})$. 


\section{Self-duality condition for U(1) symmetry:}

Let us next consider gauge fields in a four dimensional space with signature $(2,2)$. Thus, we can identify $x^{0}=t, x^{1}=x, x^{2}=\tilde{t}, x^{3}=\tilde{x}$. If we choose

$$
\epsilon^{0123}=1
$$

then it follows that in this space we will have

$$
\epsilon_{0123}=1
$$

The self-duality condition for the field strengths associated with any group are given by

$$
F_{\mu \nu}=\frac{1}{2} \epsilon_{\mu \nu}^{\lambda \rho} F_{\lambda \rho} \quad \mu, \nu, \lambda, \rho=0,1,2,3
$$

where for matrix gauge potentials, the field strength is, in general, defined to be

$$
F_{\mu \nu}=\partial_{\mu} A_{\nu}-\partial_{\nu} A_{\mu}+\left[A_{\mu}, A_{\nu}\right]
$$

For an Abelian gauge field, the self-duality relations can be written out in detail as

$$
\begin{aligned}
F_{01} & =-F_{23} \\
\text { or, } \quad \partial_{0} A_{1}-\partial_{1} A_{0} & =-\left(\partial_{2} A_{3}-\partial_{3} A_{2}\right) \\
F_{02}= & -F_{13} \\
\text { or, } \quad \partial_{0} A_{2}-\partial_{2} A_{0} & =-\left(\partial_{1} A_{3}-\partial_{3} A_{1}\right) \\
F_{03} & =-F_{12} \\
\text { or, } \quad \partial_{0} A_{3}-\partial_{3} A_{0} & =-\left(\partial_{1} A_{2}-\partial_{2} A_{1}\right)
\end{aligned}
$$

If we now assume that all the gauge potentials are independent of the extra coordinates $\left(x^{2}, x^{3}\right)$ and identify $A_{2}=A_{3}$, then the self-duality conditions in Eqs. (9)-(11) reduce to

$$
\begin{aligned}
& \partial_{0} A_{1}-\partial_{1} A_{0}=0 \\
& \left(\partial_{0}+\partial_{1}\right) A_{2}=0
\end{aligned}
$$


The solution to Eq. (13) is quite simple, namely,

$$
A_{2}(x, t)=A_{2}(t-x)
$$

It is also clear that with the choice in Eq. (4) for the gauge potentials $A_{0}$ and $A_{1}$, we obtain the KdV hierarchy from Eq. (12). This shows how the entire KdV hierarchy can be obtained from the self-duality condition on an Abelian gauge field in four dimensions.

For completeness and ease of comparison with other references, let us note that the self-duality condition as given in refs. [5] and [7] take the following form for an Abelian field.

$$
\begin{aligned}
& \partial_{t} Q-\partial_{x} H=0 \\
& \partial_{x}(Q-P)=0
\end{aligned}
$$

In this case, if we identify

$$
\begin{aligned}
& Q=P=u \\
& H=\left(-2 \lambda^{2}+\frac{1}{2} \partial^{-1}\left(\partial^{3}+2(\partial u+u \partial)\right)\right) C(u)
\end{aligned}
$$

then Eq. (16) is automatically satisfied while Eq. (15) leads to the KdV hierarchy.

\section{Self-duality condition for $\operatorname{SL}(2, R)$ symmetry:}

Let us consider again the four dimensional space with $(2,2)$ signature but gauge fields

belonging to the group $\mathrm{SL}(2, \mathbf{R})$. In this case, the self-duality conditions in Eq. (8) can be written out explicitly as

$$
\begin{aligned}
F_{01} & =-F_{23} \\
\text { or, } \quad \partial_{0} A_{1}-\partial_{1} A_{0}+\left[A_{0}, A_{1}\right] & =-\left(\partial_{2} A_{3}-\partial_{3} A_{2}+\left[A_{2}, A_{3}\right]\right) \\
F_{02}= & -F_{13} \\
\text { or, } \quad \partial_{0} A_{2}-\partial_{2} A_{0}+\left[A_{0}, A_{2}\right] & =-\left(\partial_{1} A_{3}-\partial_{3} A_{1}+\left[A_{1}, A_{3}\right]\right) \\
F_{03} & =-F_{12}
\end{aligned}
$$




$$
\text { or, } \quad \partial_{0} A_{3}-\partial_{3} A_{0}+\left[A_{0}, A_{3}\right]=-\left(\partial_{1} A_{3}-\partial_{2} A_{1}+\left[A_{1}, A_{2}\right]\right)
$$

Here we assume the gauge potentials as belonging to the $2 \times 2$ matrix representation of $\mathrm{SL}(2, \mathbf{R})$.

Once again, let us assume that all the gauge potentials are independent of the additional coordinates $\left(x^{2}, x^{3}\right)$. Furthermore, if we identify

$$
A_{2}(x, t)=A_{3}(x, t)
$$

the the self-duality conditions of Eqs. (18)-(20) reduce to

$$
\begin{aligned}
& \partial_{0} A_{1}-\partial_{1} A_{0}+\left[A_{0}, A_{1}\right]=0 \\
& \left(\partial_{0}+\partial_{1}\right) A_{2}+\left[A_{0}+A_{1}, A_{2}\right]=0
\end{aligned}
$$

If we now choose the $\operatorname{SL}(2, \mathbf{R})$ potentials as

$$
\begin{aligned}
& A_{1}=\left(\begin{array}{cc}
\lambda & -u \\
1 & -\lambda
\end{array}\right) \\
& A_{0}=\left(\begin{array}{cc}
\lambda C(u)-\frac{1}{2} \partial C(u) & -\frac{1}{2} \partial^{2} C(u)+\lambda \partial C(u)-u C(u) \\
C(u) & -\lambda C(u)+\frac{1}{2} \partial C(u)
\end{array}\right)
\end{aligned}
$$

then Eq. (22) will give the equation generating the KdV hierarchy, namely,

$$
\frac{\partial u}{\partial t}=-2 \lambda^{2} \partial C(u)+\frac{1}{2}\left(\partial^{3}+2(\partial u+u \partial)\right) C(u)
$$

It is also easy to solve Eq. (23) at least formally as

$$
A_{2}\left(x^{+}, x^{-}\right)=A_{3}\left(x^{+}, x^{-}\right)=e^{-\int^{x^{+}} d x^{\prime+} A_{+}\left(x^{\prime+}, x^{-}\right)} A_{2}\left(0, x^{-}\right) e^{\int^{+}} d x^{\prime \prime+} A_{+}\left(x^{\prime \prime+}, x^{-}\right)
$$

where we have defined

$$
\begin{aligned}
& x^{ \pm}=t \pm x \\
& A_{+}=A_{0}+A_{1}
\end{aligned}
$$

Note that the solution in Eq. (26) holds for any nontrivial matrix function $A_{2}\left(0, x^{-}\right)$. In particular, we may choose it to be a constant matrix belonging to $\mathrm{SL}(2, \mathbf{R})$. It is clear that 
any choice for $A_{2}\left(0, x^{-}\right)$does not effect the dynamical equation in Eq. (25). This shows, therefore, that the $\mathrm{KdV}$ hierarchy as well as the recursion relations can also be obtained from the self-duality condition imposed on $\operatorname{SL}(2, \mathbf{R})$ valued gauge fields in four dimensions with $(2,2)$ signature.

\section{Conclusion:}

We have given a derivation of the KdV hierarchy based on the zero curvature condition associated with a $\mathrm{U}(1)$ symmetry group. We have also obtained the KdV hierarchy from a self-duality condition on gauge fields in four dimensions (with $(2,2)$ signature) belonging to $\mathrm{U}(1)$ as well as to $\mathrm{SL}(2, \mathbf{R})$ symmetry groups. The extension of our results to other 1+1 dimensional integrable systems should be quite straightforward.

This work was supported in part by the U. S. Department of Energy Grant No. DE-FG02-91ER40685 and by CNPq, Brazil. 


\section{References:}

1. L. D. Faddeev and L. A. Takhtajan, "Hamiltonian methods in the theory of solitons", Springer (Berlin 1987).

2. A. Das, "Integrable Models", World Scientific (Singapore 1989).

3. R. S. Ward, Nucl. Phys. B236 (1984) 381; Philos. Trans. R. Soc. A315 (1985) 451; also in "Field theory, quantum gravity and strings", vol. 2, eds. H. J. deVega and N. Sanchez, p106.

4. L. J. Mason and G. A. J. Sparling, Phys. Lett. A137 (1989) 29.

5. I. Bakas and D. A. Depireux, Intern. J. Mod. Phys. A7 (1992) 1767.

6. B. B. Kadomtsev and V. I. Petviashvili, Sov. Phys. Dokl. 15 (1971) 539.

7. A. Das, Z. Khviengia and E. Sezgin, Phys. Lett. B289 (1992) 347.

8. S. S. Chern and C-K. Peng, Manuscripta Mathematica 28 (1979) 207. 